\title{
CORRIGENDUM
}

\section{Proposal for superscript diacritics for prenasalization, preglottalization and preaspiration - CORRIGENDUM}

Patricia Keating, Daniel Wymark \& Ryan Sharif

Department of Linguistics, UCLA keating@humnet.ucla.edu; dwymark@ucla.edu; ryanshari@me.com

doi:10.1017/S0025100319000057, published online by Cambridge University Press, 27 May 2019.

In the JIPA article 'Proposal for superscript diacritics for prenasalization, preglottalization and preaspiration', Figure 1 includes a typo. The diacritics for Advanced Tongue Root and Retracted Tongue Root were confused. (This error is not relevant for the content of the paper.) The correct figure is as follows:

\begin{tabular}{|c|c|c|c|c|c|c|c|c|}
\hline 8 & Voiceless & $\mathrm{n}_{\mathrm{o}} \mathrm{d}_{\mathrm{o}}$ & \& & Breathy voiced & $\mathrm{b} \quad \mathrm{a}$ & g & Dental & $\underset{n}{\mathrm{t}} \mathrm{d}_{\mathrm{d}}^{\mathrm{d}}$ \\
\hline ? & Voiced & $\stackrel{s}{t}$ & \& & Creaky voiced & $\underset{\sim}{\mathrm{b}} \underset{\sim}{\mathrm{a}}$ & e & Linguolabial & $\underset{n}{t} \quad \underset{n}{d}$ \\
\hline $\mathrm{h}^{\mathrm{h}}$ & (Post) Aspirated & $\mathrm{t}^{\mathrm{h}} \mathrm{d}^{\mathrm{h}}$ & $P_{\text {e }}$ & Preglottalized & ${ }^{3} \mathrm{t} \quad{ }^{\mathrm{P}} \mathrm{n}$ & g & Apical & $\underset{\mathrm{t}}{\mathrm{d}}$ \\
\hline $\mathrm{h}_{\mathrm{O}}$ & Preaspirated & $\mathrm{h}_{\mathrm{t}}$ & $\mathrm{w}$ & Labialized & $\mathrm{t}^{\mathrm{w}} \mathrm{d}^{\mathrm{w}}$ & 8 & Laminal & $\mathrm{t} \quad \mathrm{d}$ \\
\hline , & More rounded & P, & j & Palatalized & $t^{j} \quad t^{j}$ & थ & Rhoticity & $\partial^{2} a^{2}$ \\
\hline 8 & Less rounded & $\underset{c}{\partial}$ & i & Velarized & $t^{x} d^{X}$ & $\sim_{0}$ & Nasalized & $\tilde{\mathrm{e}}$ \\
\hline \& & Advanced & $\underset{+}{u}$ & i & Pharyngealized & $t^{\mathrm{S}} \mathrm{d}^{\mathrm{S}}$ & $\mathrm{n}_{\mathrm{O}}$ & Prenasalized & $\mathrm{n} d$ \\
\hline - & Retracted & $\underline{\mathrm{e}}$ & $\theta$ & Velarized or pharyngealized & 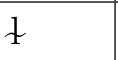 & $\mathrm{n}^{\mathrm{n}}$ & Nasal release & $\mathrm{d}^{\mathrm{n}}$ \\
\hline . & Centralized & $\ddot{\mathrm{e}}$ & G & Advanced Tongue Root & $\underset{-1}{e}$ & l & Lateral release & $\mathrm{d}^{\mathrm{l}}$ \\
\hline ; & Mid-centralized & è & P & Retracted Tongue Root & $\underset{r}{e}$ & $\bar{l}^{7}$ & No audible release & $d^{\urcorner}$ \\
\hline Y & Syllabic & $\mathrm{n}_{1}$ & I & Raised & \multicolumn{4}{|c|}{$\underset{\perp}{\mathrm{e}} \quad\left(I_{\perp}=\right.$ voiced alveolar fricative $)$} \\
\hline 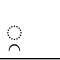 & Non-syllabic & $\stackrel{\mathrm{e}}{n}$ & ? & Lowered & \multicolumn{4}{|c|}{$\underset{T}{\mathrm{e}} \quad(\underset{T}{\beta}=$ voiced bilabial approximant $)$} \\
\hline
\end{tabular}

Figure 1 Proposed changes to IPA Diacritics chart. 


\section{Reference}

Patricia Keating, Daniel Wymark \& Ryan Sharif. Proposal for superscript diacritics for prenasalization, preglottalization and preaspiration. Journal of the International Phonetic Association, $16 \mathrm{pp}$. doi:10.1017/S0025100319000057, published online by Cambridge University Press, 27 May 2019. 